Scientific Visualization, 2021, volume 13, number 3, pages 58 - 65, DOI: 10.26583/sv.13.3.06

\title{
Study of The Cold Test Effect on Microcirculation by Video Capillaroscopy
}

\author{
M.V. Volkov,A, A.S. Machikhin²,B, E.D. Lovchikova3,B,C, D.D. Khokhlov4,B, \\ I.A. Balandin ${ }^{5, B}$, A.V. Potemkin ${ }^{6, A}$, V.S. Galanova7,B,C, I.V. Danilycheva ${ }^{8, D}$, I.V. Dorofeeva9,D \\ A University ITMO \\ в Scientific and Technological Center of Unique Instrumentation RAS \\ C Bauman Moscow State Technical University \\ D Institute of Immunology FMBA \\ ${ }^{1}$ ORCID: 0ooo-0oo2-9658-9467, ph-m.volkov@yandex.ru \\ 2 ORCID: 0000-0002-2864-3214, machikhin@ntcup.ru \\ 3 ORCID: oooo-0002-8024-1028, kat.lovchikova@yandex.ru \\ 4 ORCID: 000o-0003-0919-7762, khokhlov.dd@ntcup.ru \\ 5 ORCID: 0000-0003-2120-0186, bia5310@yandex.ru \\ 6 ORCID: 0ooo-0002-9838-2267, appanpotemkin@gmail.com \\ 7 ORCID: 0000-0002-9070-8568, victoriagalanova@icloud.com \\ 8 ORCID: 000o-0o02-8279-2173, ivdanilycheva@mail.ru \\ 9 ORCID: 0000-0002-4423-1797, idorofeeva1@gmail.com
}

\begin{abstract}
The cold stress is one of the main functional tests in the study of the microvascular bed of the skin. An important task is to automate visualization of the capillary network and measurement of microcirculation parameters directly in the local area of inflammation. In this paper, we describe a system developed for non-invasive visualization of the microcirculation and the processing of such images in order to determine the morphological parameters of the capillary network and their changes under external conditions. Proposed approach provides high-quality visualization of the skin microvessels and allows obtaining reliable information about the capillary map. The results of experimental studies are presented. Obtained data may be used for the development of the new methods for detecting hidden hemodynamic disorders and diagnostics of various diseases.

Keywords: capillaroscopy, computational imaging, microcirculation visualization, nondestructive diagnosis.
\end{abstract}

\section{Introduction}

Currently, there is an increasing interest in the study of microcirculation, which is quite understandable, since the pathology of the capillary network, which provides metabolism in tissues, is the basis of many pathological conditions [1,2]. Diseases of various etiologies can affect the skin microcirculation and lead to inflammation. Capillaries, which are the smallest and most numerous blood vessels in the human body, respond to various pathologies much earlier than arteries and veins, changes in which indicate that the disease is already in the development stage. Accurate monitoring of the capillary system functioning often allows in vivo detection of the disease at an early stage.

To put it more broadly than is customary, microcirculation includes all the processes of blood transportation through microvessels (up to 100 microns in diameter) and the exchange of fluids and substances with tissues. These two processes are extremely difficult to separate from each other. It is the state of the capillary network that provides either normal or pathological metabolism in tissues. 
In recent years, the pathogenesis of cardiovascular diseases which notoriously continue to hold the first place in mortality among the working-age population in the world according to WHO statistics, has been significantly revised. The dominant theory is the cardiovascular continuum, which describes the continuity of the development of cardiovascular system diseases from the beginning of exposure to risk factors to the death of the patient [3], where vascular damage is the link between endothelial pathology and direct tissue damage (heart attack, stroke). Previously, much attention was paid to the assessment of vasomotor activity and remodeling of large arteries of elastic and muscular type, which remains relevant today. In recent years, special attention has been paid to changes in microcirculation, suggesting that it is one of the universal development mechanisms of cardiovascular diseases.

To identify hidden hemodynamic disorders and assess possible reactions of the capillary network to external provocative effects, various functional load tests have been widely used, such as an occlusive test that assesses the amount of blood flow in the absence of arterial inflow and the reserve capabilities of the capillary network, a respiratory test that allows to assess the reactivity of the vascular wall due to the activation of the sympathetic link of the autonomous nervous system, which leads to spasm of the bringing microvessels and a temporary decrease in blood flow, postural, assessing the venoarteriolar reaction in the capillary network, thermal, etc. Of particular interest in the study of microcirculation are the cold pressor and cold local tests, carried out in the form of either immersion of the feet and / or hands of the subject in cold water (pressor), or applying a test tube with ice to the test area for 15 minutes (local). The local cold test is not as indicative in terms of changes in the capillary network as the pressor but allows it to be carried out by a larger number of subjects due to less aggressive exposure.

\section{Cold test}

During the study, attention is usually paid to the color of the background (pale, pink, red, cyanotic, cloudy), the number of capillaries, the width of their lumen, the shape, length and width of the loops, the nature of the blood flow (continuous, fast, slow, intermittent), the vulnerability of the capillary wall (hemorrhages, rupture of individual capillaries) $[4,5]$. The normal picture of capillaries is characterized by a pale pink or pink background, the number of capillary loops is up to 10-12 in $1 \mathrm{~mm}$, the shape resembles the heads of hairpins arranged in the form of a palisade, the blood flow is continuous and fast. Each loop consists of a narrow arterial and a wider venous knee. Spasm is characterized by a significant decrease or disappearance of the arterial branch. With atony, there is a sharp expansion of both branches. In spastic atony, both changes are present simultaneously.

Samples with the influence of aggressive environmental factors that can affect the microcirculation are used to detect early changes in the capillary network. In this case, accordingly, there may be an adequate or pathological reaction of the microcirculatory bed. As one of these factors, cold exposure in the form of a cold sample is used. Cold exposure in any case causes changes in the picture of the microcirculatory bed, which can be observed using the capillaroscopy method. The question is how adequately the microcirculation vessels react to the cold and how quickly the normal blood flow and the state of the vascular wall are restored.

When cooling the human skin below $15^{\circ} \mathrm{C}$, temperature reduction is combined with vasoconstriction of arteries, resistive microvessels, and arteriolo-venous anastomoses [6]. However, after a few minutes (usually 5-10 minutes with a cold pressor test or faster with local cooling), there is an increase in the local skin temperature and vascular dilation (cold vasodilation reaction). The cause of cold vasodilation is cold paralysis of the leiomyocytes of the vascular wall, especially arteriolo-venous anastomoses, as a result of which (with the most pronounced reaction) they lose the ability to contract, expand and turn into a passive vascular bed. As the temperature increases due to the heating of the blood vessel walls, supplied in a large volume, the ability to reduce them is restored, which leads to vasoconstriction. Such 
alternating episodes of constriction-dilation can be repeated during prolonged cooling. Since the cold vasodilation reaction can be observed in areas of denervated skin, it is considered a local phenomenon. Cold dilatation is most pronounced in areas of the skin with the presence of arteriolo-venous anastomoses (finger pads, nail bed), where it is advisable to carry out cold test since it is the most informative area. However, since the reaction of cold vasodilation can be observed (although not constantly) in areas of the skin that do not contain arteriolovenous anastomoses (forearm, back of the hand and foot, hip, etc.), the study can be carried out here if necessary. Many authors regard cold vasodilation as an adaptive-protective reaction to cold stress. There is information that with an organic lesion of the vascular wall (late organic stage of Raynaud's disease, for example) this reaction is lost. From these positions, the safety and severity of cold vasodilation can be considered as one of the criteria for compensating the functional resources of tissue microcirculation in conditions of pathology.

Thus, the safety and severity of vasodilation in a cold skin test, evaluated using the capillaroscopy method, can be an indicator of the safety or, conversely, pathology of the capillary network, particularly in the early stages of diseases affecting the microcirculation when the changes are still reversible and functional in nature. Tracking the slightest changes in the blood vessels will help patients avoid the serious consequences of diseases, such as ulcers and necrosis.

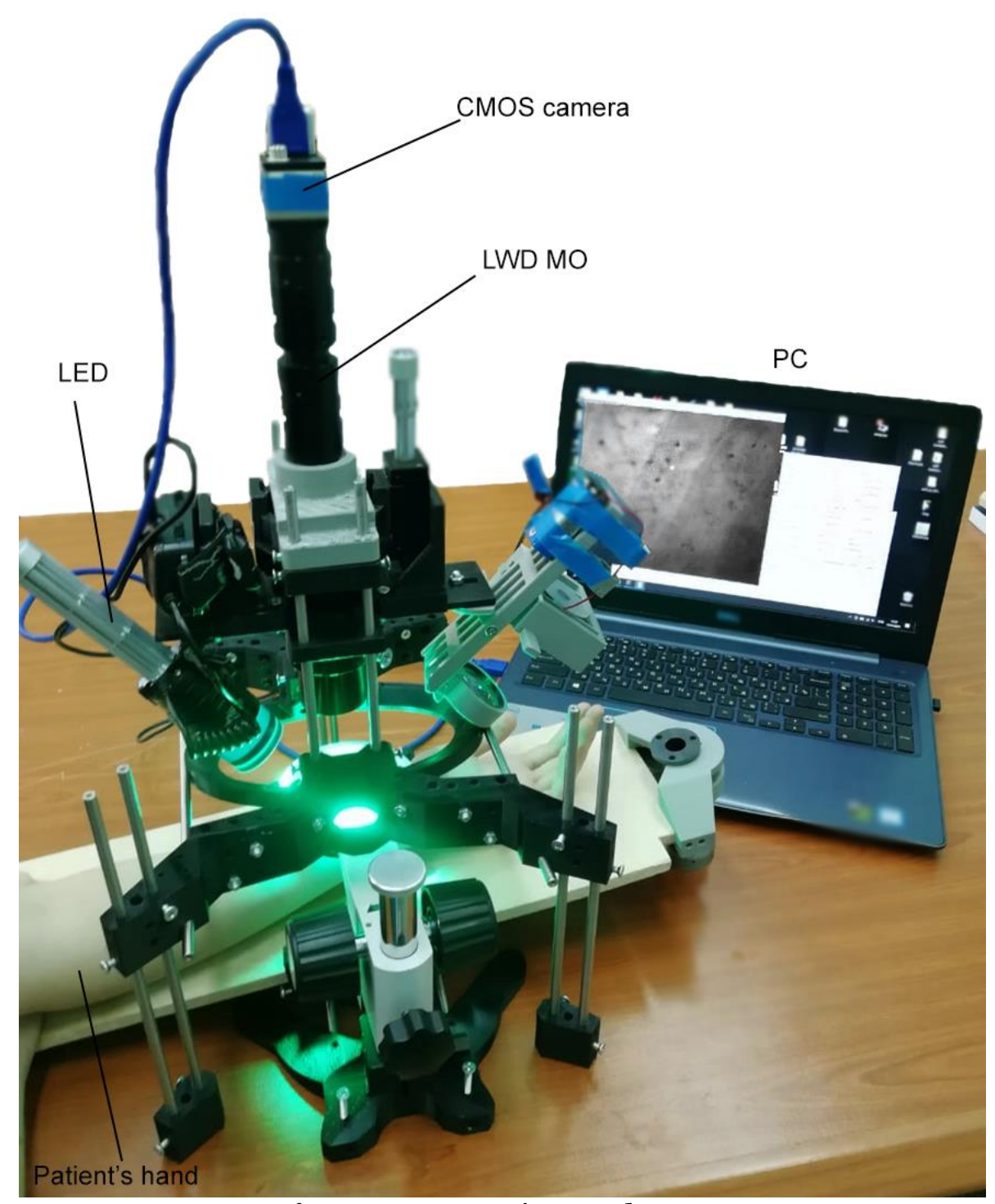

Figure 1. Experimental setup. 


\section{Experimental setup}

Experimental setup is illustrated in Figure 1. During image acquisition, patient keeps his hand on an adjustable wooden mount. To flatten the inspected skin region in the forearm area and to get rid of glare from the epidermis upper layer, the thin glass plate treated with oil covers the skin. Illumination system is based on a powerful green LED with lens focusing light onto the specimen. Image acquisition system includes long working distance $4.5 \mathrm{x}$ microscope objective (LWD MO) and monochrome CMOS camera (1936 × 1216 pixels, 5.9 $\mu \mathrm{m} \times 5.9 \mu \mathrm{m}$ pixel pitch). They are both attached to a $z$-stage providing accurate focusing. This setup provides high quality images of the small skin areas with close-to-micron resolution. The linear field of view is $1.5 \mathrm{~mm} \times 1.5 \mathrm{~mm}$.

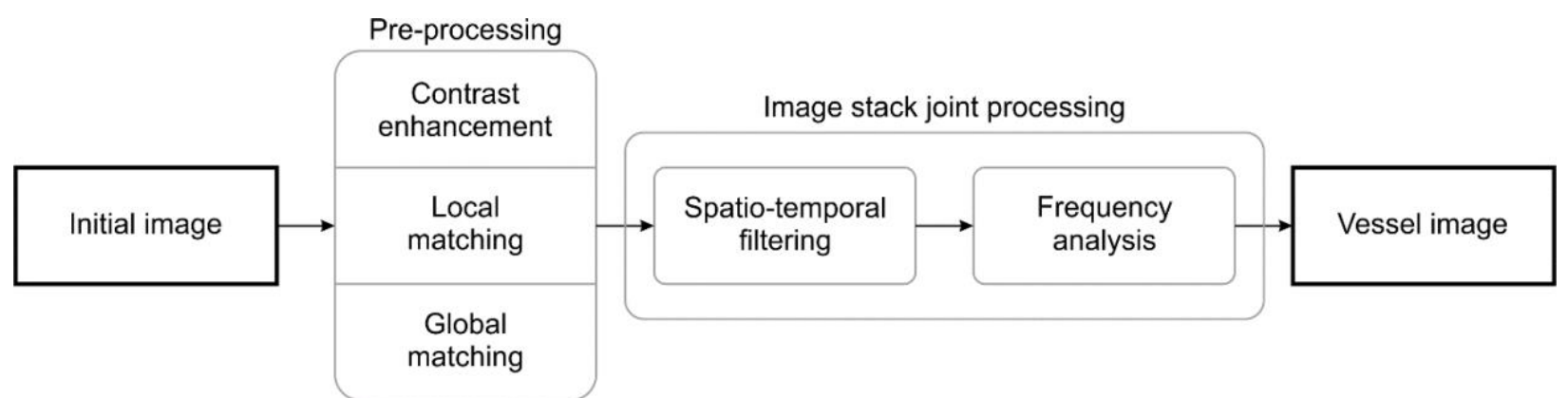

Figure 2. Image processing pipeline.

The image processing pipeline is presented in Figure 2 and described in detail in $[7,8]$. Figure 3 illustrates the images obtained at different stages of the pipeline. Before the joint processing of image stack the initial images acquired by the camera (Figure 3a), preprocessing is necessary in order to eliminate the influence of uneven illumination distribution across the field of view, body vibrations and patient breathing. It includes contrast enhancement, illumination non-uniformity correction, global and local matching [9]. We implement contrast enhancement by normalizing initial image intensities using a preliminary calibration image of a uniform test-object. Exclusion of the low-frequency components from each image of the stack and intensity normalization allow illumination non-uniformity correction. Local matching procedure includes evaluation of local motion vectors between the consequent images in the stack and image matching with respect to the directions and lengths of these vectors. The common area of overlapped images is cropped after local matching. Thus, we obtain the intensity-corrected, stabilized and aligned image stack (Figure 3b).
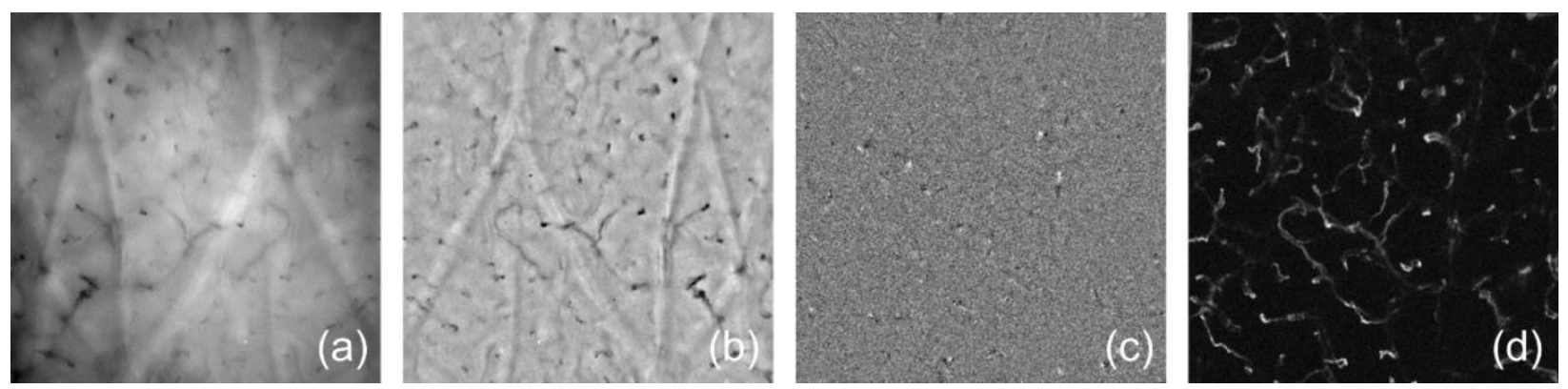

Figure 3. Initial (a), pre-processed (b), blood flow (c) and vessel (d) images.

After pre-processing, the image stack is treated as a spatiotemporal data cube $I_{k}(x, y)$. Each image pixel of this cube contains temporal dependences of the signal $I_{k}=I\left(t_{k}\right)$ reflected from the particular points of the inspected skin area. The vessels may be distinguished from the surrounding tissues by the presence of blood flow changes. Spectral analysis of the temporal signal $I\left(t_{k}\right)$ using Fourier transform allows detection of the dominant frequencies corresponding to the blood flow. After we obtain the intensity deviation 
values in each pixel, we eliminate the background, which is free from blood flow, and continue processing only the image pixels with significant intensity oscillations in the frequency range of $0.5-20 \mathrm{~Hz}$ to calculate blood flow images (figure $3 \mathrm{c}$ ). The intensities of blood volume changes represented as the ratio of high- and low-frequency spectral components in the Fourier spectrum allow vessel image calculation (Figure 3d).

\section{Results}

The described setup was implemented for the analysis of forearm areas of 5 healthy people. To provide an adequate comparison of the inspected skin areas before and after cold stress, we ensured their exact match by selecting the areas with specific features (birthmarks, scars, etc.). Each experiment included the acquisition of image stacks containing 3000 images of $1000 \times 1000$ pixels resolution obtained at $50 \mathrm{~Hz}$ camera frame rate and processing according to the algorithm described above. The image stacks were obtained before and 5 minutes after the cold stress, carried out by applying a glass flask with ice to the selected skin area for 10 minutes. Figure 4 shows one of the acquired images (male, 33 years) and calculated vessel maps before and after cold stress. The changes in microcirculation may be clearly noticed by the reduction of active capillaries amount.
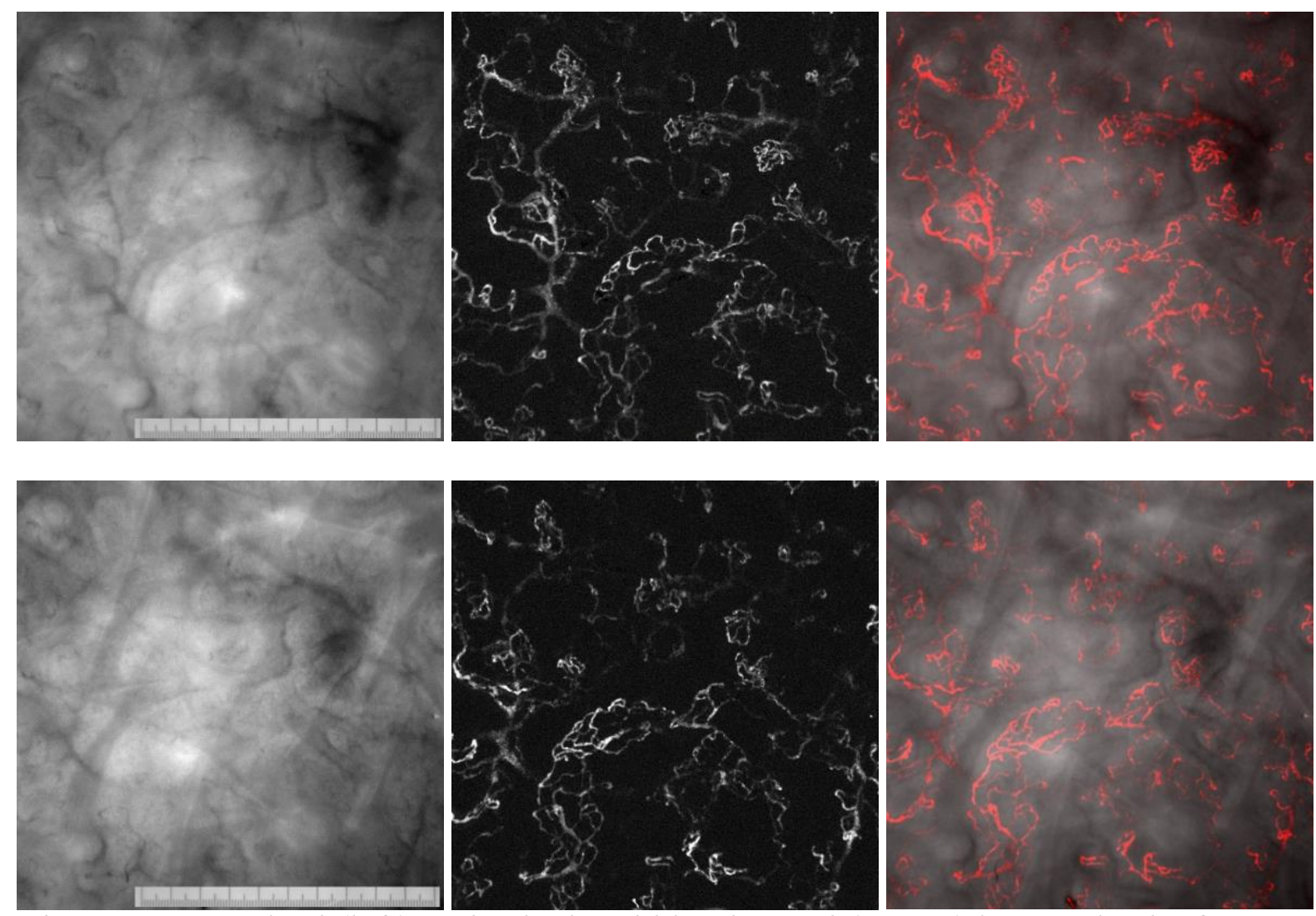

Figure 4. Acquired (left) and calculated blood vessel (center) images in the forearm area before (upper row) and after (lower row) cold stress and their overlap (right).

An important feature provided by the proposed technique is photoplethysmogram acquisition. It is widely in use for detection and quantitative analysis of volumetric changes in blood circulation, estimating oxygen saturation, measuring blood pressure and other physiological parameters. This technique has also become a powerful tool for analyzing the effect of the external stressors on microcirculation, including the cold test [10]. Being based on monitoring the changes in the intensity of light reflected from the skin, it may be easily implemented by the processing of obtained images. After the temporal dependence of integral intensity is calculated and aligned, we may obtain a photoplethysmogram shown in Figure 5 and measured from the same data as presented in Figure 4. The frequency of the pulses 
indicates the heartbeat. The pulse shape is usually associated with the state of tissue and its pathologies. We may notice that cold stress changes the amplitude of the pulses, introduces its temporal instability and makes the pulse shape more rugged.

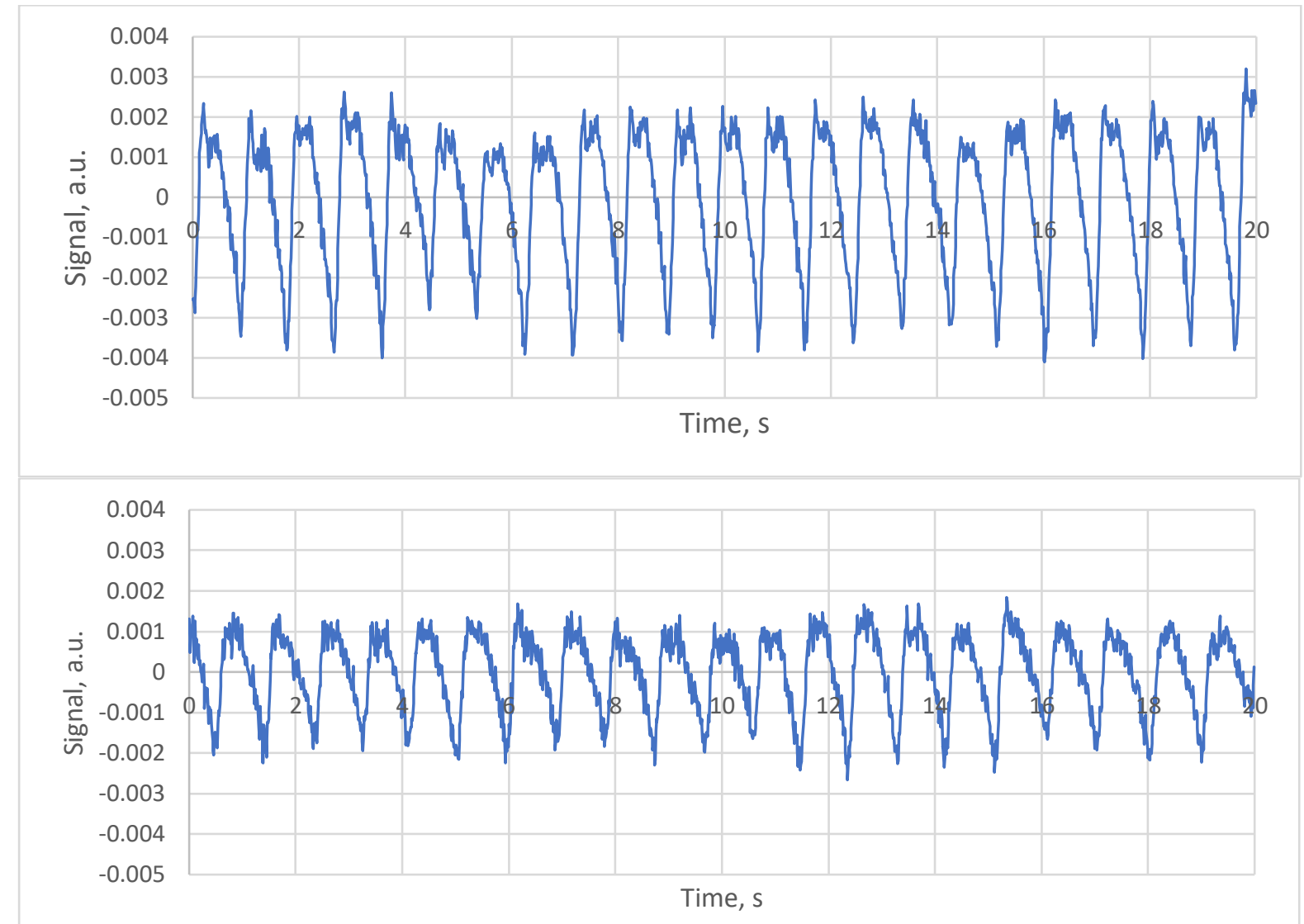

Figure 5. Calculated photoplethysmogram in the forearm area before (upper) and after (low) cold stress.

\section{Conclusion}

In this study, we have demonstrated that video capillaroscopy may become an effective tool for microcirculation analysis under external stress. It provides non-invasive mapping of microvessels activity and assessment of the morphology (quantity, density, shape, dimensions) of capillaries in the forearm area. Proposed setup is quite easy-to-adjust, enables fast and high-quality skin image acquisition and allows applying video capillaroscopy and photoplethysmography methods. To shed the light on the mechanism of vessels activation (or deactivation) after cold stress, more capillaroscopic experiments accompanied with measurement of skin temperature, blood pressure and other parameters have to be carried out. Further research may also include quantitative study of blood flow parameters and mapping the blood flow velocities across the whole area inspected.

\section{Acknowledgements}

This study is supported by RF President Grant (project MD-32.2021.4).

\section{References}

[1] Guven G., Hilty P. M. and Ince C. Microcirculation: Physiology, Pathophysiology, and Clinical Application / Blood Purification, 2020, Vol. 49, pp. 143-150. 
[2] Tafner P.F.D.A., Chen F.K., Rabello Filho R., Corrêa T.D., Chaves R.C.D.F. and Serpa Neto A. Recent advances in bedside microcirculation assessment in critically ill patients / Revista Brasileira de Terapia Intensiva, 2017, Vol. 29, pp. 238-247.

[3] Camici P. G. and Magnoni M. How important is microcirculation in clinical practice? / European Heart Journal Supplements, 2019, Vol. 21, No. Supplement_B, pp. B25-B27.

[4] Naidu S., Baskerville P. A., Goss D. E. and Roberts V. C. Raynaud's phenomenon and cold stress testing: a new approach / European Journal of Vascular Surgery, 1994, Vol. 8, No. 5, pp. 567-573.

[5] Colfer H. T., Das S. K., Dabich L., Randall O. S. and Pitt B. Effect of cold stress on coronary sinus blood flow in patients with scleroderma / Journal of the Association for Academic Minority Physicians, 1993, Vol. 4, No. 2, pp. 62-65.

[6] Novikova I. N., Dunaev A. V., Sidorov V. V. and Krupatkin A. I. Possibilities of cold test to functional estimation of microcirculatory-tissue systems / Regional blood circulation and microcirculation, 2015, Vol. 14, No. 2, pp. 47-55.

[7] Volkov M. V., Margaryants N. B., Potemkin A. V., Machikhin A. S., Khokhlov D. D., Batshev V. I., Danilycheva I. V. and Danilychev M. V. Blood vessel visualization method in human skin based on video recording of blood flow using a laparoscope / Journal of Communications Technology and Electronics, 2020, Vol. 65, No. 7, pp. 806-814.

[8] Machikhin A. S., Volkov M. V., Khokhlov D. D., Lovchikova E. D., Potemkin A. V., Danilycheva I. V., Dorofeeva I. V. and Shulzhenko A. E. Exoscope-based videocapillaroscopy system for in vivo skin microcirculation imaging of various body areas / Biomedical Optics Express, 2021, Vol. 12, No. 8, pp. 4627-4636.

[9] Volkov M. V., Margaryants N. B., Potemkin A. V. and Gurov I. P. The method of compensation for local displacements of images of capillaries in the evaluation of capillary blood flow parameters / Journal of Physics: Conference Series, 2019, Vol. 1421, No. 012054.

[10] Budidha K. and Kyriacou P. A. Photoplethysmography for quantitative assessment of sympathetic nerve activity (SNA) during cold stress / Frontiers in Physiology, 2019, Vol. 9, p. 1863. 